\title{
Theoretical Investigation of Correlation, Quantum Electrodynamics, and Breit Effects on the Energy Levels of Ca-Like Tungsten
}

\author{
G.G. KOnAN* AND L. ÖZDEMİR \\ Department of Physics, Sakarya University, 54187 Sakarya, Turkey \\ (Received March 15, 2019; revised version May 10, 2019; in final form December 9, 2019)
}

\begin{abstract}
We have investigated the electron correlation effects, the Breit interaction, and quantum electrodynamics effects on the energy levels of Ca-like tungsten $\left(\mathrm{W}^{54+}\right.$ ) using AUTOSTRUCTURE code developed by Badnell. These effects are of great importance in heavy highly charged ions. In this work, calculated results have been discussed and compared with other available results in literature.
\end{abstract}

DOI: 10.12693/APhysPolA.137.289

PACS/topics: correlations, energy levels, Breit effects, QED

\section{Introduction}

Highly charged heavy elements and ions are of interest in atomic calculations. Atomic tungsten is a high- $Z$ element, and of particular importance for providing tests of quantum electrodynamics (QED) in the region of a very strong Coulomb field of the nucleus [1]. In addition, the relativistic effects depend strongly on $Z$ and are important $[2,3]$ besides correlation effects. Also, atomic data of tungsten ions (W, $Z=74)$ are very important in astrophysics and plasma physics.

Theoretical calculations and experimental measurements on various highly ionized Ca-like tungsten ion were reported. Theoretically, the energy levels and spectral lines of multiply ionized tungsten atoms, $\mathrm{W}^{2+}$ through $\mathrm{W}^{73+}$ including Ca-like tungsten were compiled by Kramida and Shirai [4]. Safronova and Safronova [5] tabulated wavelengths and transition probabilities for $n l-n^{\prime} l^{\prime}$ transitions in $\mathrm{W}^{54+}$ ion. Wavelengths and transition probabilities were calculated for forbidden lines of $3 d^{2}$ ground configurations of $\mathrm{W}^{54+}$ ion by Quinet [6]. Electron impact excitation and polarization studies of Ca-like $\mathrm{W}^{54+}$ ion were presented by Dipti et al. [7]. Guo et al. [8] reported relativistic many-body calculations on energy levels, wavelengths, and transition probabilities for forbidden transitions within the ground configurations in Ca-like tungsten. Fischer et al. [9] computed energy levels of the $3 d^{2}$ configurations for $\mathrm{W}^{54+}$ ion. Extensive self-consistent multi configuration Dirac-HartreeFock (MCDHF) calculations were performed for the $3 d^{2}$ ground configurations of $\mathrm{W}^{54+}$ ion by Zhao et al. [10]. Finally, Ding et al. calculated energy levels and radiative transitions for $\mathrm{W}^{54+}$ ion comprehensively [11-14].

*corresponding author; e-mail: ggunday@sakarya.edu.tr
On the experimental side, Ralchenko et al. $[15,16]$ measured extreme ultraviolet (EUV) spectra of highly charged tungsten ions, including $\mathrm{W}^{54+}$ ion, using an electron-beam ion trap (EBIT). Lennartsson et al. [17] observed the $E 1$ spectrum of the excited state $[\mathrm{Ne}] 3 s^{2} 3 p^{5} 3 d^{3}$ to the ground state [Ne] $3 s^{2} 3 p^{6} 3 d^{2}$ of $\mathrm{W}^{54+}$ ion measured between 27 and $41 \AA$ at the Livermore EBIT-I electron-beam ion trap using a high-resolution grazing-incidence spectrometer.

In this paper, the energy levels of $\mathrm{W}^{54+}$ ion have been studied first using AUTOSTRUCTURE code developed by Badnell [18]. Then, the Breit interaction (magnetic interaction between the electrons and retardation effects of the electron-electron interaction) and QED (self-energy and vacuum polarization) contributions have been investigated. Two different configuration sets for calculations have been studied to investigate the impact of valence-valence correlation $(\mathrm{VV})$ and core-core correlation $(\mathrm{CC})$ within the framework of configuration interaction (CI) expansion: $3 d n l(n=3-5$ and $l=0-4)$, $4 \ln l(n=4-5$ and $l=0-4)$, outside the core $1 s^{2} 2 s^{2} 2 p^{6} 3 s^{2} 3 p^{6}$ for valence-valence correlation, and $3 p^{6} 3 d 4 l(l=0-3), 3 p^{6} 4 l 4 l^{\prime} \quad\left(l=0-1\right.$ and $\left.l^{\prime}=0-3\right)$, $3 p^{6} 3 d 5 s, 3 p^{6} 3 d 5 p, 3 p^{6} 4 d^{2}, 3 p^{6} 5 s^{2}, 3 p^{6} 5 s 5 p, 3 p^{6} 5 s 5 d$, $3 p^{5} 3 d^{3}, \quad 3 p^{5} 3 d^{2} 4 s, \quad 3 p^{5} 3 d 4 s 4 d, \quad 3 p^{5} 4 s^{2} 4 p, \quad 3 p^{5} 4 s 4 p^{2}$, $3 p^{5} 4 p^{3}, 3 p^{5} 4 s^{2} 4 d, 3 p^{5} 3 d 4 s 4 p, 3 p^{4} 3 d^{3} 4 s$ outside the core $1 s^{2} 2 s^{2} 2 p^{6} 3 s^{2}$ for core-core correlation.

\section{Calculation method}

AUTOSTRUCTURE code $[18,19]$ is a general program for the calculation of atomic and ionic energy levels, radiative, and autoionization rates and photoionization cross-sections using non-relativistic or semirelativistic wave functions. It is based on SUPERSTRUCTURE [20]. In this code, the configuration set is chosen optionally and added new configuration to improve accuracy (a configuration interaction 
expansion, CI expansion). The CI expansion is related to the choice of radial functions. Each $(n l)$ radial function is calculated in the Thomas-Fermi or Slater-type-orbital potential model. The Hamiltonian in any coupling model (LS, IC, or ICR) is diagonalized to obtain eigenvalues and eigenvectors with which to construct the rates. This code makes use of non-relativistic or kappa-averaged relativistic wave functions and the full Breit interaction in the Pauli approximation.

Both of QED and Breit relativistic effects are added as a perturbation correction. Quantum electrodynamics contributions consist of self energy and vacuum polarization contributions to level energies. The finite-nucleus effect is taken into account by assuming an extended Fermi distribution for the nucleus. Orbitals are fixed, but the mixing coefficients are obtained by diagonalizing the modified Hamiltonian. CI method was used to account for the correlation effects.

The details of the method can be found in Refs. $[18,19,21]$ and we only summarize this here briefly. For an ion with $N$ electrons, a set of configurations

$$
c=\prod_{n l}(n l)^{q_{n l}}, \quad \sum_{i} q_{i}=N
$$

defines a trial solution $\Psi(\gamma)$ to a suitable Hamiltonian $H$ in the multiconfigurational sum form

$$
\Psi\left(\gamma \mid \boldsymbol{x}_{1}, \ldots \boldsymbol{x}_{N}\right)=\sum_{k} a_{k} \Phi_{k}\left(c_{k}(\gamma) \mid \boldsymbol{x}_{1}, \ldots \boldsymbol{x}_{N}\right),
$$

where $\gamma$ denotes configuration and coupling scheme. In intermediate coupling (IC) wave functions can be written as

$$
\Psi=\Psi\left(\Gamma S L J M_{J} \mid \boldsymbol{x}_{1}, \ldots \boldsymbol{x}_{N}\right),
$$

which are eigenvectors to the Breit-Pauli matrix $\left\langle k\left|H_{B P}\right| k^{\prime}\right\rangle$ with eigenvalues $E_{k}$.

\section{Results and discussion}

In this study, we have presented the energy levels for $\mathrm{W}^{54+}$ using AUTOSTRUCTURE code developed by Badnell [18]. Also the purpose of the present work is to investigate the effects of QED, Breit, and correlations on the energy levels. Relativistic and QED effects depend strongly on $Z[2,3]$, and therefore it is important to include for atomic modelling in highly charged ions such as Ca-like tungsten. In addition, the electron correlation effects due to the Coulomb interaction between the electrons are also important.

We have examined the effects of valence-correlation and core-core correlation on the energy levels by choosing two different configuration sets. In calculation VV, only valence correlation is considered while core-core (CC) electron correlation contributions are considered with single and double excitations from $3 \mathrm{p}$. We have focused on different $\mathrm{VV}$ and $\mathrm{CC}$ configuration sets. In the first step, we have studied minimum configurations. Then more configurations have been inserted the configuration set. Although, only the best results of them have been presented and compared. We have taken into account the configurations of $3 d n l$ ( $n=3-5$ and $l=0-4), 4 \ln l(n=4-5$ and $l=0-4)$, outside the core $1 s^{2} 2 s^{2} 2 p^{6} 3 s^{2} 3 p^{6}$ for valence-valence (VV) correlation, and $3 p^{6} 3 d 4 l(l=0-3), 3 p^{6} 4 l 4 l^{\prime} \quad\left(l=0-1\right.$ and $\left.l^{\prime}=0-3\right)$, $3 p^{6} 3 d 5 s, 3 p^{6} 3 d 5 p, 3 p^{6} 4 d^{2}, 3 p^{6} 5 s^{2}, 3 p^{6} 5 s 5 p, 3 p^{6} 5 s 5 d$, $3 p^{5} 3 d^{3}, \quad 3 p^{5} 3 d^{2} 4 s, \quad 3 p^{5} 3 d 4 s 4 d, \quad 3 p^{5} 4 s^{2} 4 p, \quad 3 p^{5} 4 s 4 p^{2}$, $3 p^{5} 4 p^{3}, 3 p^{5} 4 s^{2} 4 d, 3 p^{5} 3 d 4 s 4 p, 3 p^{4} 3 d^{3} 4 s$ outside the core $1 s^{2} 2 s^{2} 2 p^{6} 3 s^{2}$ for core-core (CC) correlation.

Table I displays the present energy levels and comparison with other available works. The AUTOSTRUCTURE energy levels are generated in $\mathrm{cm}^{-1}$. But we have converted the energy values to $\mathrm{eV}$ in order to simplify the comparison with other works $\left(1 \mathrm{eV}=8065.44 \mathrm{~cm}^{-1}\right)$. The correlation effects (valence-valence, $\mathrm{VV}$, and corecore, CC) and the Breit and QED contributions on energy levels are exhibited in this table. We have omitted the core $1 s^{2} 2 s^{2} 2 p^{6} 3 s^{2} 3 p^{6}$ in the table. We have obtained 453 energy levels from VV correlation and 1275 energy levels from CC. Here we have only listed the levels of $n l$ $(n=3-4, l=0-2)$.

In Table II, the results for the energies of the $3 d^{2}$ states are compared with Ding et al. [14] to find out which values are better. We have obtained better results studying with CC instead of VV. Also we can see that, after considering the Breit and QED corrections, the present results are closer to the comparing values. The differences of $\mathrm{CC}+$ Breit $+\mathrm{QED}$ results are smaller than the others in all levels except $3 d^{2}$ ${ }^{3} F_{2}$. The differences (\%) may be up to 3.360 for $\mathrm{VV}$, 2.923 for $\mathrm{VV}+$ Breit $+\mathrm{QED}, 2.730$ for $\mathrm{CV}$, and 2.307 for $\mathrm{CV}+$ Breit $+\mathrm{QED}$. We can say that the calculated results using the configuration set including electron excitations from $3 p$ subshell (core-core correlation) and taking Breit and QED contributions $(\mathrm{CC}+$ Breit $+\mathrm{QED})$ are better, in general. These $\mathrm{CC}+\mathrm{Breit}+\mathrm{QED}$ results are found to be in good agreement with other works in the literature $[5,6,8-10,14,22]$. It should be emphasized that electron correlation, QED and the Breit relativistic contributions should be added in the calculations to obtain accurate results. These contributions hold significance in particular for highly charged ions. We have calculated $\left(\left|E_{\text {our }}-E_{\text {other }}\right| / E_{\text {other }}\right) \times 100$; the differences in percent, for the accuracy of our $\mathrm{CC}+$ Breit + QED results according to Ding et al. [14] and Fig. 1 shows the differences for all $3 d^{2}$ and $3 d^{3}$ levels. We note that there are small differences in the energies in comparison with results reported by Ding et al. [14]. The differences $(\%)$ are less than 2.5 for $3 d^{2}$ levels and less than 3.5 for $3 d^{3}$ levels.

The results obtained in this work are systematically higher than the ones available in the literature. We could not clearly explain this case. But, we think that this case occurs from the difference of used methods. We calculated present results with AUTOSTRUCTURE which uses non-relativistic or semi-relativistic wave functions. Although, comparing values are from fully relativistic methods like MCDF. 
Energy levels, $E$ (in eV), for Ca-like tungsten $\left(\mathrm{W}^{54+}\right)$

TABLE I

\begin{tabular}{|c|c|c|c|c|c|c|}
\hline \multirow{2}{*}{\multicolumn{2}{|c|}{ Levels }} & \multicolumn{4}{|c|}{ This work } & \multirow{2}{*}{ Other works } \\
\hline & & $\mathrm{VV}$ & $\mathrm{VV}+$ Breit+QED & $\mathrm{CC}$ & $\mathrm{CC}+$ Breit $+\mathrm{QED}$ & \\
\hline $3 d^{2}$ & ${ }^{3} F_{2}$ & 0.000 & 0.000 & 0.000 & 0.000 & $0.000^{a, b}$ \\
\hline $3 d^{2}$ & ${ }^{3} P_{0}$ & 22.918 & 22.901 & 22.899 & 22.883 & $23.3^{a}, 23.123^{b}, 23.173^{c}, 23.199^{d}$ \\
\hline $3 d^{2}$ & ${ }^{3} F_{3}$ & 74.073 & 73.711 & 73.570 & 73.221 & $\begin{array}{l}22.920^{e}, 24.322^{f}, 23.045^{g}, 23.089^{h} \\
72.590^{a}, 72.456^{b}, 72.345^{c}, 72.264^{d},\end{array}$ \\
\hline $3 d^{2}$ & ${ }^{3} P_{2}$ & 84.234 & 83.872 & 83.742 & 83.393 & $\begin{array}{l}72.630^{e}, 72.009^{f}, 72.484^{g}, 72.499^{h} \\
82.882^{a}, 82.805^{b}, 82.772^{c}, 82.600^{d},\end{array}$ \\
\hline $3 d^{2}$ & ${ }^{3} P_{1}$ & 88.753 & 88.385 & 88.262 & 87.907 & $\begin{array}{l}82.822^{e}, 83.076^{f}, 82.784^{g}, 82.816^{h} \\
87.9^{a}, 87.613^{b}, 87.669^{c}, 87.460^{d}\end{array}$ \\
\hline $3 d^{2}$ & ${ }^{1} G_{4}$ & 89.260 & 88.883 & 88.716 & 88.351 & $\begin{array}{l}87.629^{e}, 88.160^{f}, 87.608^{g}, 87.625^{h} \\
86.4^{a}, 86.358^{b}, 86.429^{c}, 86.021^{d}, \\
86.385^{e}, 86.128^{f}, 86.306^{g}, 86.305^{h}\end{array}$ \\
\hline $3 d^{2}$ & ${ }^{3} F_{4}$ & 156.914 & 156.189 & 155.896 & 155.196 & $153.0^{a}, 153.158^{b}, 153.009^{c}, 152.704^{d}$ \\
\hline $3 d^{2}$ & ${ }^{1} D_{2}$ & 164.347 & 163.617 & 163.356 & 162.651 & $\begin{array}{l}153.369^{e}, 152.591^{f}, 153.146^{g}, 153.191^{h} \\
161.1^{a}, 161.160^{b}, 161.006^{c}, 160.774^{d},\end{array}$ \\
\hline $3 d^{2}$ & ${ }^{1} S_{0}$ & 188.926 & 188.183 & 188.028 & 187.311 & $\begin{array}{l}161.214^{e}, 161.397^{f}, 161.115^{g}, 161.201^{h} \\
185.1^{a}, 185.139^{b}, 184.860^{c}, 184.927^{d}, \\
184.883^{e}, 186.772^{f}, 184.946^{g}, 185.196^{h}\end{array}$ \\
\hline $3 d 4 s$ & ${ }^{3} D_{1}$ & 2086.340 & 2085.242 & 2051.203 & 2050.737 & - \\
\hline $3 d 4 s$ & ${ }^{3} D_{2}$ & 2087.497 & 2086.397 & 2052.365 & 2051.898 & - \\
\hline $3 d 4 p$ & ${ }^{3} F_{2}$ & 2163.144 & 2162.820 & 2129.556 & 2128.736 & - \\
\hline $3 d 4 s$ & ${ }^{3} D_{3}$ & 2164.797 & 2163.330 & 2131.640 & 2130.815 & - \\
\hline $3 d 4 p$ & ${ }^{3} D_{1}$ & 2166.872 & 2165.404 & 2161.572 & 2161.255 & - \\
\hline $3 d 4 s$ & ${ }^{1} D_{2}$ & 2166.876 & 2166.542 & 2165.269 & 2164.947 & - \\
\hline $3 d 4 p$ & ${ }^{1} D_{2}$ & 2243.250 & 2242.557 & 2240.904 & 2240.231 & - \\
\hline $3 d 4 p$ & ${ }^{1} F_{3}$ & 2244.332 & 2243.637 & 2242.368 & 2241.694 & - \\
\hline $3 d 4 p$ & ${ }^{3} D_{2}$ & 2297.002 & 2295.625 & 2294.704 & 2293.334 & - \\
\hline $3 d 4 p$ & ${ }^{3} P_{1}$ & 2300.618 & 2299.253 & 2298.163 & 2296.804 & - \\
\hline $3 d 4 p$ & ${ }^{3} F_{3}$ & 2301.124 & 2299.755 & 2299.091 & 2297.731 & - \\
\hline $3 d 4 p$ & ${ }^{3} P_{0}$ & 2302.788 & 2301.432 & 2300.422 & 2299.072 & - \\
\hline $3 d 4 p$ & ${ }^{3} P_{2}$ & 2376.940 & 2375.203 & 2373.758 & 2372.041 & - \\
\hline $3 d 4 p$ & ${ }^{3} F_{4}$ & 2377.144 & 2375.411 & 2374.466 & 2372.753 & - \\
\hline $3 d 4 p$ & ${ }^{3} D_{3}$ & 2377.606 & 2375.860 & 2374.831 & 2373.108 & - \\
\hline $3 d 4 p$ & ${ }^{1} P_{1}$ & 2383.961 & 2382.229 & 2381.117 & 2379.406 & - \\
\hline $3 d 4 d$ & ${ }^{3} D_{1}$ & 2417.458 & 2415.892 & 2418.680 & 2417.102 & - \\
\hline $3 d 4 d$ & ${ }^{3} G_{3}$ & 2417.596 & 2416.028 & 2418.819 & 2417.240 & - \\
\hline $3 d 4 d$ & ${ }^{3} F_{2}$ & 2428.155 & 2426.624 & 2428.767 & 2427.228 & - \\
\hline $3 d 4 d$ & ${ }^{3} P_{0}$ & 2439.719 & 2438.201 & 2440.107 & 2438.585 & - \\
\hline $3 d 4 d$ & ${ }^{1} F_{3}$ & 2448.819 & 2447.151 & 2448.850 & 2447.187 & - \\
\hline $3 d 4 d$ & ${ }^{3} D_{2}$ & 2451.272 & 2449.607 & 2451.313 & 2449.652 & - \\
\hline $3 d 4 d$ & ${ }^{3} G_{4}$ & 2453.447 & 2451.781 & 2453.473 & 2451.812 & - \\
\hline $3 d 4 d$ & ${ }^{3} S_{1}$ & 2454.980 & 2453.325 & 2454.984 & 2453.334 & - \\
\hline $3 d 4 d$ & ${ }^{3} F_{3}$ & 2500.039 & 2498.126 & 2500.317 & 2498.408 & - \\
\hline $3 d 4 d$ & ${ }^{1} D_{2}$ & 2503.595 & 2501.694 & 2503.777 & 2501.879 & - \\
\hline $3 d 4 d$ & ${ }^{1} G_{4}$ & 2505.758 & 2503.856 & 2505.869 & 2503.970 & - \\
\hline
\end{tabular}

${ }^{a}$ Ref. [22], ${ }^{b}$ Ref. [14], ${ }^{c}$ Ref. [6], ${ }^{d}$ Ref. [5], ${ }^{e, f}$ Ref. [8], ${ }^{g}$ Ref. [10], ${ }^{h}$ Ref. [9] 


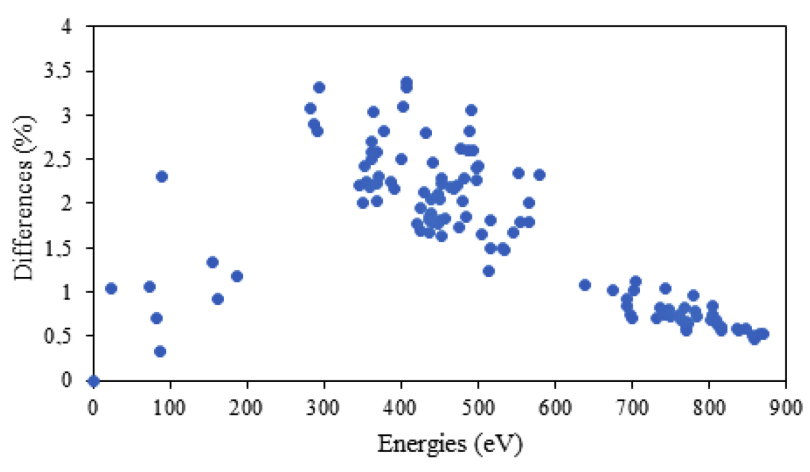

Fig. 1. Differences in percent between present AUTOSTRUCTURE results and MCDF [14].

TABLE II

Differences for energy levels of this work with [14] for $\mathrm{W}^{54+}$

\begin{tabular}{cc|c|c|c|c}
\hline \hline Levels & VV & $\begin{array}{c}\text { VV+Breit } \\
+ \text { QED }\end{array}$ & CC & $\begin{array}{c}\text { CC+Breit } \\
+ \text { QED }\end{array}$ \\
\hline $3 d^{2}$ & ${ }^{3} F_{2}$ & 0.000 & 0.000 & 0.000 & 0.000 \\
$3 d^{2}$ & ${ }^{3} P_{0}$ & 0.886 & 0.960 & 0.968 & 1.037 \\
$3 d^{2}$ & ${ }^{3} F_{3}$ & 2.231 & 1.732 & 1.537 & 1.055 \\
$3 d^{2}$ & ${ }^{3} P_{2}$ & 1.725 & 1.288 & 1.131 & 0.710 \\
$3 d^{2}$ & ${ }^{3} P_{1}$ & 1.301 & 0.881 & 0.740 & 0.335 \\
$3 d^{2}$ & ${ }^{1} G_{4}$ & 3.360 & 2.923 & 2.730 & 2.307 \\
$3 d^{2}$ & ${ }^{3} F_{4}$ & 2.452 & 1.979 & 1.787 & 1.330 \\
$3 d^{2}$ & ${ }^{1} D_{2}$ & 1.977 & 1.520 & 1.362 & 0.925 \\
$3 d^{2}$ & ${ }^{1} S_{0}$ & 2.045 & 1.644 & 1.560 & 1.173
\end{tabular}

\section{Conclusion}

A systematic study is carried out to investigate the effect of electron correlation, quantum electrodynamics, and the Breit relativistic contributions on the energy levels of Ca-like W. We show four different results for energies including different effects. When we compared our AUTOSTRUCTURE results with available theoretical and experimental data, a good agreement was observed. It is found that quantum electrodynamics, the Breit relativistic contributions, and configuration interaction effects play important roles on energy levels of highly ionized atoms. Hence, we hope that these present AUTOSTRUCTURE results will be useful for experimental researches in the future.

\section{References}

[1] A.N. Artemyev, V.M. Shabaev, V.A. Yerokhin, G. Plunien, G. Soff, Phys. Rev. A 71, 062104 (2005).

[2] J.D. Gillaspy, J. Phys. B 34, R93 (2001).

[3] I. Martinson, Nucl. Instrum. Methods Phys. Res. B 43, 323 (1989).

[4] A.E. Kramida, T. Shirai, At. Data Nucl. Data Tables 95, 305 (2009).

[5] U.I. Safronova, A.S. Safronova, J. Phys. B 43, 074026 (2010).

[6] P. Quinet, J. Phys. B 44, 195007 (2011).

[7] T.D. Dipti, L. Sharma, R. Srivastava, Can. J. Phys. 93, $888(2015)$.

[8] X.L. Guo, M. Huang, J. Yan, S. Li, R. Si, C.Y. Li, C.Y. Chen, Y.S. Wang, Y.M. Zou, J. Phys. B 48, 144020 (2015).

[9] C.F. Fischer, G. Gaigalas, P. Jönsson, Atoms 5, 7 (2017).

[10] Z.L. Zhao, K. Wang, S. Li, R. Si, C.Y. Chen, Z.B. Chen, J. Yan, Y. Ralchenko, At. Data Nucl. Data Tables 119, 314 (2018).

[11] X. Ding, R. Sun, J. Liu, F. Koike, I. Murakami, D. Kato, H.A. Sakaue, N. Nakamura, C. Dong, J. Phys. B 50, 045004 (2017).

[12] X. Ding, R. Sun, F. Koike, D. Kato, I. Murakami, H.A. Sakaue, C. Dong, Eur. Phys. J. D 71, 73 (2017).

[13] X. Ding, J. Yang, F. Koike, I. Murakami, D. Kato, H.A. Sakaue, N. Nakamura, C. Dong, J. Quantitat. Spectrosc. Radiat. Transf. 204, 7 (2018).

[14] X. Ding, R. Suna, F. Koike, I. Murakami, D. Kato, H.A. Sakaue, N. Nakamura, C. Dong, At. Data Nucl. Data Tables 119, 354 (2018).

[15] Y. Ralchenko, I.N. Draganic, J.N. Tan, J.D. Gillaspy, J.M. Pomeroy, J. Reader, U. Feldman, G.E. Holland, J. Phys. B 41, 021003 (2008).

[16] Y. Ralchenko, I.N. Draganic, D. Osin, J.D. Gillaspy, J. Reader, Phys. Rev. A 83, 032517 (2011).

[17] T. Lennartsson, J. Clementson, P. Beiersdorfer, Phys. Rev. A 87, 062505 (2013).

[18] N.R. Badnell, Comput. Phys. Commun. 182, 1528 (2011).

[19] N.R. Badnell, J. Phys. B 19, 3827 (1986).

[20] W. Eissner, M. Jones, H. Nussbaumer, Comput. Phys. Commun. 8, 270 (1974).

[21] N.R. Badnell, J. Phys. B 30, 1 (1997).

[22] NIST Atomic Spectra Database Levels Data. 